\title{
The jitter in stimulated orbicularis oculi muscle: technique and normal values
}

\author{
J V TRONTELJ, A KHURAIBET,* M MIHELIN
}

From the University Institute of Clinical Neurophysiology, University Medical Centre of Ljubljana, Yugoslavia

SUMMARY A technique is described of measuring the motor end plate jitter in the orbicularis oculi muscle activated by extra-muscular nerve stimulation, standardised for routine use in diagnosis and evaluation of neuromuscular transmission disorders. Among the advantages of the technique are comparatively quick and easy sampling of adequate number of single motor end-plates, convenient control of discharge rate between less than 1 and up to $20 \mathrm{~Hz}$, absence of direct muscle fibre responses, and little discomfort for the patient. The jitter in the orbicularis oculi is significantly lower than that in the extensor digitorum communis muscle. There is a positive correlation between the jitter and the latency of the individual muscle fibre responses, possibly indicating that smaller diameter muscle fibres have slightly lower safety factor of neuromuscular transmission. The data collected in a group of healthy volunteers suggest the following upper normal limits for the mean of absolute consecutive difference of the latency: $30 \mu$ s for individual motor end plates (one out of 20 values may be higher), and $18 \mu$ s for a median of 20 motor end plates.

Single fibre electromyography (SFEMG) is a sensitive diagnostic method for neuromuscular transmission disorders, ${ }^{12}$ and its diagnostic yield in myasthenia gravis is significantly higher than that of repetitive nerve stimulation and determination of acetylcholine receptor antibodies. ${ }^{3}$ In a recent study of 450 myasthenic patients, ${ }^{3}$ the jitter was abnormal in $95 \%$, when two muscles were studied. In patients with moderate to severe generalised symptoms, abnormal findings were obtained in $100 \%$, and in those with mild generalised weakness in $95 \%$. However, in patients with the ocular form of myasthenia and in those in clinical remission the jitter was only abnormal in $61 \%$ and $51 \%$, respectively. A facial muscle (frontalis) had a higher diagnostic yield than limb muscles (extensor digitorum communis), the jitter being more abnormal in the former in $72 \%$ of 156 patients compared to $17 \%$ with more pronounced abnormality in the latter. This is consistent with earlier observations. $^{145}$ In our experience with a small series of patients with mild generalised disease and patients in

*Visiting Research Fellow, Department of Clinical Neurophysiology, Ibn Sina Hospital, Kuwait.

Address for reprint requests: Prof Jože Trontelj, University Institute of Clinical Neurophysiology, Zaloška 7, 61105 Ljubljana, Yugoslavia.

Received 8 January 1988. Accepted 10 February 1988 remission, orbicularis oculi muscle was more often abnormal than the frontalis muscle (Trontelj, unpublished material). Unfortunately it is not the easiest muscle to study. Some patients find it difficult to maintain uniform contraction at the desired level at which individual motor units can be studied in satise⿳⺈, factory separation from each other. By estimating the jitter during extramuscular nerve stimulation as described in the present report this difficulty can be avoided. The purpose of this work was to standardise the technique and collect normal values.

\section{Subjects and methods}

Subjects Nineteen healthy subjects agreed to participate in the study. Their ages ranged from 19 to 62 years, mean 34.5 years, SD 10.2 years. There were nine females and 10 males with similar mean ages (33.0 and 35.9 years, respectively). Stimulation With the subject in the supine position, a pencil-shaped surface stimulation cathode with a blunt point was used to find a low-threshold stimulation site where the facial nerve branches to the orbicularis oculi traverse the zygomatic arch (fig 1). Stimulation at this site produced small twitches in only the lateral quadrant of the orbital portion of the muscle and, occasionally, in the upper or lower palpebra. Then a needle cathode (Teca MF37 Teflon coated monopolar steel needle insulated to $1 \mathrm{~mm}$ from the tip) was inserted at this point subcutaneously and its position was adjusted while stimulating at $2 \mathrm{~Hz}$ so as to obtain a reasonably low threshold for visible twitches and to have stimulation pain- 


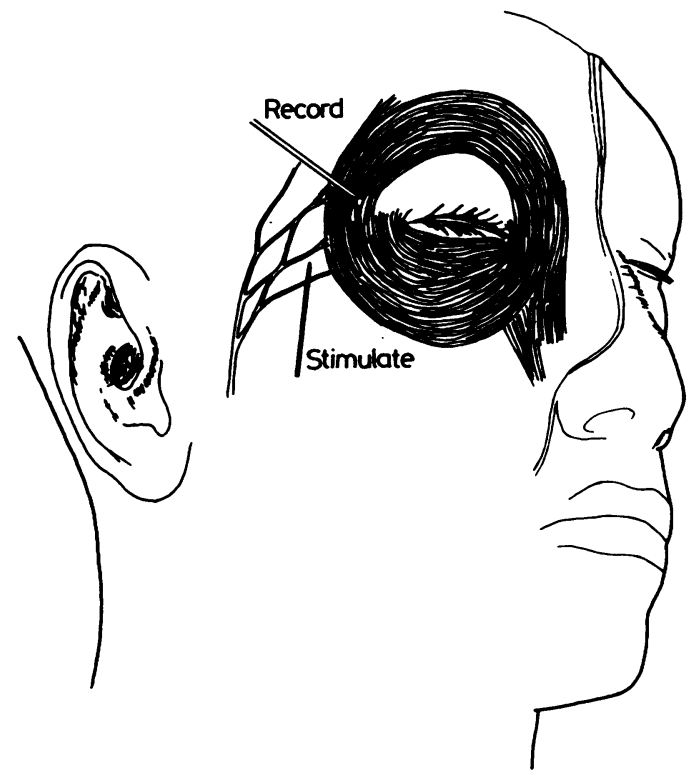

Fig 1 The approximate position of the stimulating and recording electrodes. The anatomical details were redrawn from ref. 6.

less (occasionally it was moderately painful even at threshold stimulation, presumably due to vicinity of a trigeminal nerve twig; a slight change of the electrode position then made stimulation painless, even at higher stimulus intensities). A Medelec GE-V 53058 ground electrode, wrapped around the neck, served as an anode. The stimulus was a $50 \mu \mathrm{s}$ rectangular pulse from a Medelec type SC6 voltage output stimulator and an IS/V stimulus isolation unit with a 10-turn potentiometer allowing fine control of stimulus amplitude. This was considered an important advantage, as it made possible fine adjustments needed for example to differentially stimulate axons with similar thresholds. The stimulus amplitudes most commonly ranged between 30 and $60 \mathrm{~V}$. The stimulation rate was $2 \mathrm{~Hz}$ during the search for suitable positions of the stimulating and the recording electrodes and $10 \mathrm{~Hz}$ during the jitter measurement.

Recording A Medelec SF 2553031 SFEMG electrode was inserted into the orbital portion of the orbicularis oculi muscle, about $10 \mathrm{~mm}$ lateral to the outer canthus, just at the margin of the orbit, at a distance of about 15-25 mm above and medially from the tip of the stimulating cathode (fig 1). A Medelec MS 6 electromyograph with an AA6 amplifier was used for recording. The frequency filters were set to $3.2 \mathrm{KHz}$ and $16 \mathrm{KHz}$ (in case of a low amplitude of the recorded single fibre action potential the upper filter was set to 8 or $3.2 \mathrm{KHz}$ to reduce the amplitude of the high frequency noise). The rather high setting of the lower filter served to minimise the stimulus artefact, and attenuate the undesired contribution of the more distant fibres. Discrete single fibre action potentials were isolated in the response by proper adjustments of stimulus amplitude. Complex potential shapes due to superimposition of several muscle fibres were not accepted for measurement. An exception was made when at least the complete main positive-negative slope and both peaks were uncontaminated by action potentials from other fibres, and the other action potentials riding on the leading or terminal portion of the studied spike were also stable. The criteria used to identify an action potential as being generated by a single muscle fibre were similar to those used in fibre density estimations; ${ }^{2}$ stable shape, amplitude of more than $0.2 \mathrm{mV}$ and rise time of less than $0.3 \mathrm{~ms}$. Great care was taken to ensure that during jitter measurement stimulus amplitude was well suprathreshold for the studied axon. This was achieved by identifying the threshold amplitude, and then increasing it gradually to the point beyond which there was no further latency shortening or visible reduction of the jitter. A further increment by a few volts was considered sufficient to provide a safe margin of suprathreshold stimulus strength. Occasionally, the latency of consecutive single fibre responses showed discrete jumps by about 150-200 $\mu$ s, well visible at sweep speed of $1 \mathrm{~ms}$ per division. Such alternation was considered to be due to alternation of the nodes of Ranvier from which the nerve action potential was triggered by the stimulus. Such recordings were discarded, unless further increment in stimulus strength abolished these latency shifts, stabilising the responses at the earlier of the two latencies. Longer latency jumps between 0.5 and several ms were occasionally also seen, again depending on stimulus strength. These represented axon reflexes; ${ }^{7}$ the jitter was measured either at the early or at the late latency position, whichever appeared to be more stable or less disturbed by other fibres action potentials. After having studied responses of axons with lowest threshold at a given recording position, the stimulus amplitude was gradually raised to recruit additional axons. About 5-10 muscle fibres belonging to 3-5 different axons could usually be studied from a single recording position. Then the position of the recording or that of the stimulating needle, or both, was changed, until 20-30 different muscle fibres were sampled.

Jitter measurement The latency was measured from the stimulus to a selected point on the rising (positive-negative) slope of the action potential, which was monitored at $100 \mu \mathrm{s}$ per division on a Z-modulated delayed sweep of a Tektronix 565 oscilloscope. A home-built jittermeter ${ }^{8}$ was used on-line to compute the jitter expressed as mean of absolute consecutive differences (MCD) of the latency. The resolution of the system is $0.1 \mu \mathrm{s}$, but the readings for jitter values above $5 \mu \mathrm{s}$ were made at a resolution of $1 \mu \mathrm{s}$. At least five series of 50 consecutive responses were acquired. If the results of the consecutive series of responses of a muscle fibre were not closely similar, adequacy of the stimulus was checked and additional series were acquired. The quality of recording was monitored visually on the screen; series where some of the responses were superimposed by an accidental response from another axon were naturally discarded. The latency from the stimulus was recorded for each muscle fibre. The complete recording session took 30 to 45 minutes.

Data analysis Of the five MCD values obtained for each muscle fibre, the mean of the lowest three values was computed. However, the criterion for the acceptance of a muscle fibre in the statistical material was that the range of these three values did not exceed $5 \mu \mathrm{s}$ or $20 \%$ of the mean value. If this criterion was not fulfilled all data for that muscle fibre 


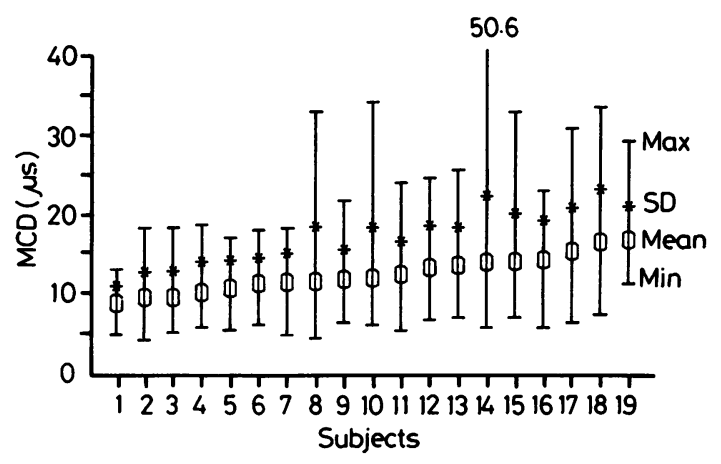

Fig 2 Mean MCD (circles), SD (between circles and asterisks) and range of $M C D$ values (between dashes) for the individual subjects, arranged according to the increasing mean $M C D$

were rejected, on the assumption that the quality of stimulation or recording was not satisfactory. Not more than one or rarely two sets of data per subject were discarded for this reason.

\section{Results}

The mean MCDs and ranges are plotted for each subject in fig 2 . As can be seen, the scattering of data is relatively small. The pooled data are shown in fig 3 , compared with similarly collected data for the extensor digitorum communis (EDC) muscle. ${ }^{9}$ The MCD values of individual muscle fibres ranged from $4.0 \mu \mathrm{s}$ to $50.6 \mu \mathrm{s}$ in the orbicularis oculi, compared with a range from 5.0 to 72.2 in the EDC muscle. The distribution histograms are slightly skew, similar for the two muscles; however, the values for the orbicularis oculi are significantly smaller. The mean MCD, computed from means of the individual subjects, was $12.4 \mu \mathrm{s}$, and the mean MCD, computed from the pooled data, was $12.4 \mu \mathrm{s}$ (table). The mean response latency was $4.8 \mathrm{~ms}$, SD $2 \cdot 5$, range $1 \cdot 1-16.5$ ms. There was a significant positive correlation between the jitter of the stimulated muscle fibres and their latency (fig 4). On the other hand, there was no correlation between any of the SFEMG parameter and the age or sex of the subjects.

The distribution of the pooled data, as well as of those from individual subjects suggests that the following upper normal limits may be used, above the 97th percentile and close to the 99th percentile: $30 \mu \mathrm{s}$ for individual muscle fibres, and $20 \mu \mathrm{s}$ for a mean of 20-25 sampled fibres. With a provision similar to those in other jitter studies, the test may be pronounced normal if no more than one out of 20 muscle fibres exceeds the individual upper normal limit of 30 $\mu \mathrm{s}$, and if the mean MCD of the remaining fibres stays below $20 \mu$ s. Both requirements were fulfilled by each of our subjects.

\section{Discussion}

The method of jitter measurement in electrically stimulated muscle has been elaborated and standardised earlier. ${ }^{9}$ Among the important advantages of this 0 form of activation of the muscle is the perfect control of the discharge rate, which minimises the "myogenic" contribution to the jitter due to the "velocity recovery function". ${ }^{10}$ Compared with the jitter study in voluntarily activated muscle, stimulation study is $\stackrel{0}{\rightarrow}$ generally faster and requires little co-operation of the $\bar{c}$ patient. The technique described in this paper differs from the previously reported method of jitter study by $\frac{\bar{c}}{\frac{}{2}}$ intramuscular axonal stimulation ${ }^{9}$ in the following $\stackrel{\mathbb{Q}}{\circ}$ points:

(1) The nerve fibres are stimulated outside the muscle. In this way direct stimulation of the muscle fibres is. avoided. This is particularly important if high resolu- $\overrightarrow{\vec{\omega}}$ tion time measurement is not available; in this case $\stackrel{\sigma}{\omega}$ low jitter of the directly stimulated muscle fibre can be mistaken for a normal reading. In the present study, the lowest MCD value in an individual series in one or fibre was $4.0 \mu \mathrm{s}$, and values between 4.0 and $5.0 \mu$. were quite rare, seen in only five out of 517 musc fibres. In the extensor digitorum communis muscle, $a+$ similar jitter study, but with intramuscular stimue lation, produced between $5 \%$ and $20 \%$ direct musc fibre responses. In that muscle, MCD value of less than $4.5 \mu$ s was considered to indicate direct musce fibre stimulation, and values between 0.8 and 3.5 , $\overrightarrow{8}$ were usually obtained in such instances when the $\infty$ recording conditions were good (even small noise such as with higher gains introduces a few $\mu \mathrm{s}$ of "technical" jitter). Motor end plate jitter lower than $4 \mu$ s has so far

Table Mean MCD, SD and range for pooked data and as computed from means of the individual subjects, compared with a previous study in the EDC muscle 9

\begin{tabular}{|c|c|c|}
\hline & Orbicularis oculi & $\begin{array}{l}\text { Extensor digitorum } \\
\text { communis }\end{array}$ \\
\hline $\begin{array}{l}\text { No. of subjects } \\
\text { No. of muscle fibres } \\
\text { MCD-pooled data }[\mu \mathrm{s}]\end{array}$ & $\begin{array}{r}19 \\
517\end{array}$ & $\begin{array}{r}15 \\
516\end{array}$ \\
\hline $\begin{array}{l}\text { mean } \\
\text { range } \\
97 \text { th percentile } \\
\text { mean }+3 \mathrm{SD}\end{array}$ & $\begin{array}{l}12 \cdot 4 \\
5 \cdot 6 \\
4 \cdot 0-50 \cdot 6 \\
25 \cdot 0 \\
29 \cdot 2\end{array}$ & $\begin{array}{l}17 \cdot 1 \\
8 \cdot 2 \\
5 \cdot 0-72 \cdot 2 \\
37 \cdot 0 \\
41 \cdot 7\end{array}$ \\
\hline $\begin{array}{l}\text { MCD-individual subjects } \\
\text { mean of mean MCDs } \\
\text { SD of mean MCDs } \\
\text { range of mean MCDs } \\
\text { mean }+3 \mathrm{SD}\end{array}$ & $\begin{array}{l}12 \cdot 4 \\
2 \cdot 3 \\
8 \cdot 7-16 \cdot 8 \\
19 \cdot 3\end{array}$ & $\begin{array}{l}17 \cdot 2 \\
3 \cdot 0 \\
12 \cdot 8-23 \cdot 4 \\
26 \cdot 2\end{array}$ \\
\hline $\begin{array}{l}\text { Upper normal limits } \\
\text { MCD of individual fibres } \\
\text { Mean MCD of } 20 \text { fibres }\end{array}$ & $\begin{array}{l}30^{*} \\
20\end{array}$ & $\begin{array}{l}40^{*} \\
25\end{array}$ \\
\hline
\end{tabular}

*Up to one in twenty fibres are allowed to exceed these limits. 


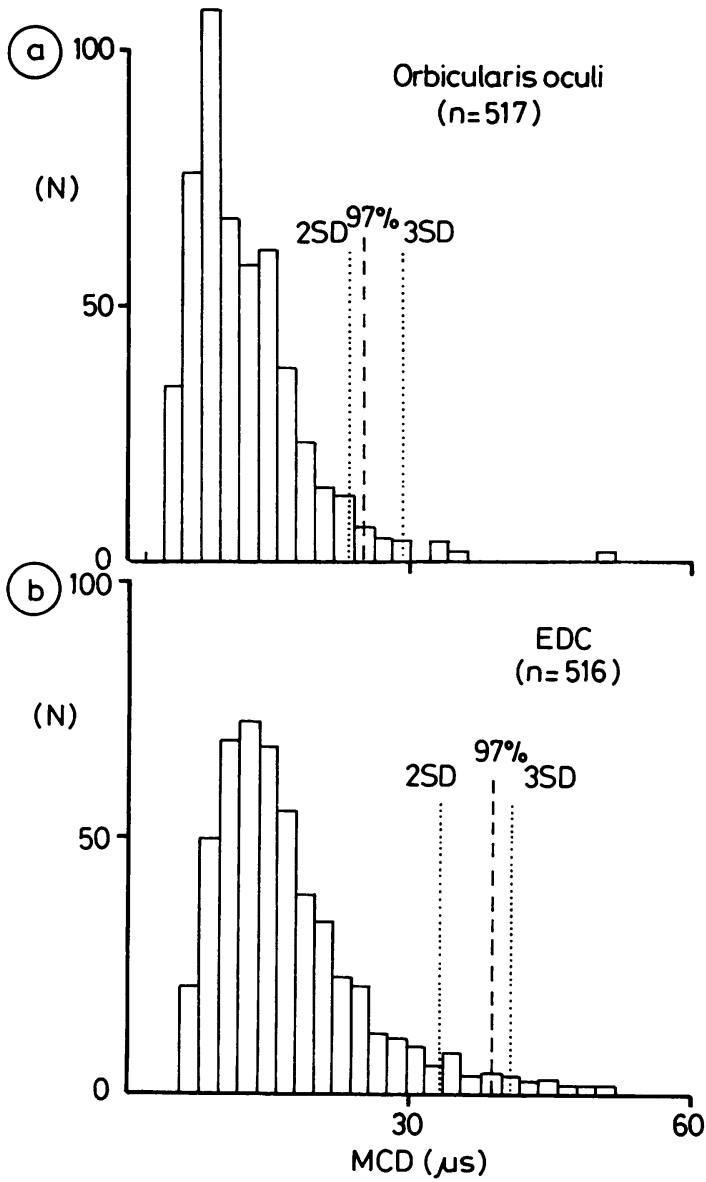

Fig 3 Pooled MCD values for all muscle fibres of all subjects (a) shown in comparison with similarly collected material from the EDC muscle $(b)$. The dotted lines indicate the mean +2 and 3 SD limits, and the 97th percentile, respectively.

not been identified in the extensor digitorum communis muscle. In a diagnostic jitter study, the ability to avoid direct muscle fibre stimulation and thus the risk of underestimating the mean MCD value is an important advantage. In the case of the extensor digitorum communis, the same could be achieved by stimulating the nerve outside the muscle; however, the finely graded "microstimulation", which is possible inside the muscle due to dispersion of the small nerve bundles, would be lost. Such fine activation is essential for good quality SFEMG recording. In the case of the orbicularis oculi, the facial nerve branch destined to it is already divided in several smaller bundles outside the muscle (cf. fig 1).

(2) The technique is applied to the orbicularis oculi muscle, which appears to be particularly sensitive in showing abnormality early in the course of generalised myasthenia gravis, as well as in the ocular form of the disease. Along with the frontalis, it may be one of the few abnormal muscles during the clinical remission. ${ }^{3}$ Since stimulation makes the testing of this muscle quick and easy for both the electromyographer and the patient, it might be recommended as the first choice, to replace the voluntary activation jitter study in the extensor digitorum communis, the deltoid or the frontalis muscles. In our hands, it is easier than intramuscular stimulation study in the extensor digitorum communis (EDC).

(3) In the orbicularis oculi muscle, with the technique described, it is easier to test different stimulation rates than in the EDC. Stimulation at $20 \mathrm{~Hz}$ commonly produced displacement of the recording electrode in the EDC, but not in the orbicularis oculi. Raising the rate from 10 to $20 \mathrm{~Hz}$ produces slight increase in the jitter in normal subjects, but may change a normal jitter in a mildly affected myasthenic patient to grossly abnormal values associated with blocking. As stimulation at this high rate is continued, the abnormality becomes progressively more prominent. Interposition of a period of rest characteristically reverses the abnormality. Both change in the discharge rate and the effect of rest intervals on the degree of the abnormal jitter and blocking are less easily tested in the voluntary activation jitter study due to frequent loss of the recording position. These two manoeuvres may additionally increase the sensitivity of the technique (Trontelj, preliminary observations) and could make the recognition of the presynaptic (Lambert-Eaton) type of abnormality at the level of individual motor end plates easier.

(4) There was a significant positive correlation between the latency of orbicularis oculi muscle fibres and the magnitude of their jitter. Longer latency can be due to slower conduction in the nerve fibre, longer neuromuscular transmission time, longer impulse pathway due to an eccentric motor end plate position, or slower conduction of the action potential in a muscle fibre with smaller diameter. Axon reflex response is an additional possible cause; however, such responses were relatively infrequent in our situation with extramuscular nerve stimulation (in contrast to intramuscular stimulation) since significant axonal branching does not occur until a few mm proximal to the entry point of the nerve into the muscle. The respective contributions to the longer latency of the mentioned factors were not studied; however, the smaller muscle fibre diameter is considered to be a major cause. The tendency of such fibres to have larger jitter may be of physiological interest in the light of the recently described relationship between the muscle fibre diameter and the motor unit size. ${ }^{11}$ 


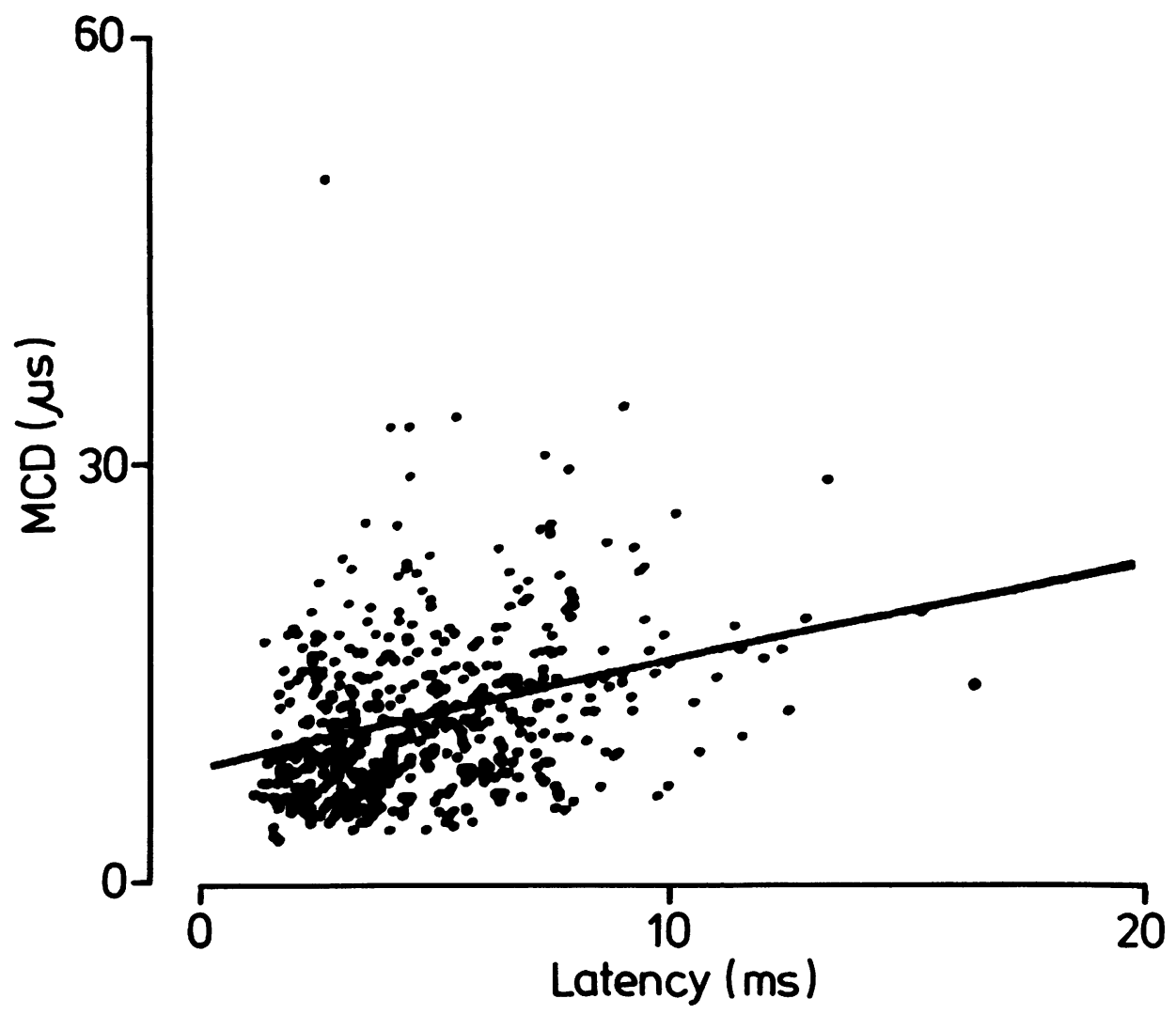

Fig 4 The jitter values plotted against the latencies of the 517 individual muscle fibres, show a significant positive correlation $(r=0.32, t=7.6, p<0.001)$.

(5) The MCD values obtained in the orbicularis oculi were $4.6 \mu$ s or $27 \%$ lower than those collected in the EDC muscle, ${ }^{9}$ the difference being significant at a level of $p<0.001(t=10.6$ for pooled data and 5.1 for means of subjects). A difference of a similar size has been found earlier between the frontalis and the EDC muscles in voluntary activation studies, ${ }^{2}$ reflecting physiological differences between the facial and the limb muscles. In the normal muscle, the jitter is correlated with the safety factor of neuromuscular transmission relative to curare. ${ }^{12}$ However, the muscles with a normally higher safety factor do not appear to be less susceptible to myasthenic involvement.

The subjects tolerated the test very well and found stimulation considerably easier than voluntary activation, quite comparable to the stimulation study in the EDC. Stimulation itself was painless in all subjects after it became clear that the position of the cathode tip was critical, the occasional pain probably being produced by accidental stimulation of a trigeminal nerve twig (one of the subjects actually experienced toothache during stimulation). Relaxation of the studied muscle was easily achieved and some of the subjects even became drowsy.

To summarise, the described technique seems to be easier and quicker and to cause less discomfort to the patient than the voluntary activation jitter study in the orbicularis oculi muscle. The mean jitter value, as well as the suggested upper normal limit in this muscle are significantly lower than in the EDC. The technique is expected to prove better suited for evaluation of patients with suspected neuromuscular transmission defect than voluntary activation jitter study or jitter studies by intramuscular axonal stimulation in some other muscles. In practice, the jitter can be well estimated from two or three series of 50 responses for each of about 20 muscle fibres, and the test can be completed in less than three quarters of an hour.

The jitter can be measured by any automatic device 
with a resolution of up to $5 \mu \mathrm{s}$, and also manually from film recordings, since there is no need to recognise low jitter, as direct muscle fibre stimulation does not occur. The suggested upper normal limits of $30 \mu \mathrm{s}$ for individual motor end plates (one in 20 may exceed this limit) and $20 \mu$ s for the mean of 20 end plates are even above 3 SD (99.7th percentile) for both values; therefore they appear to be safely high even when in the clinical setting the quality control of recording and computation is less rigid than exercised in this study. It should be pointed out, however, that these limits are only valid up to the age of 60-70 years. Above 70, a few abnormal values are not unusual, probably due to neurogenic changes. ${ }^{2}$

In clinical use, mean MCD of a sample of muscle fibres may not be an ideal measure of the jitter. Already one very abnormal motor end plate in a usual sample of 20 can push the arithmetic mean out of the normal range. The median value may be a more appropriate parameter; however, it must be used in combination with the other criterion of abnormality, that is, the number of motor end plates outside the normal range. The mean of median MCDs of normal subjects in this study was $11.4 \mu \mathrm{s}$, SD $2.1 \mu \mathrm{s}$; the upper normal limit close to mean +3 SD is estimated at $18 \mu \mathrm{s}$.

The authors gratefully acknowledge the skilful help of Miss Tatjana Gorjanc, the neurophysiological assistant. Thanks are also due to our friends and colleagues who participated as subjects. The study was financially supported by the Slovene Research Community, grant No. C3-0178-306-87

\section{References}

1 Stalberg E, Trontelj JV, Schwartz MS. Single muscle fibre recording of the jitter phenomenon in patients with myasthenia gravis and in members of their families. Ann NY Acad Sci 1976;274:189-262.

2 Stalberg E, Trontelj JV. Single Fibre Electromyography. Old Woking, Surrey: Mirvalle Press, 1979:1-244.

3 Sanders D, Howard JF Jr. Single-fiber electromyography in myasthenia gravis. AAEE minimonograph No. 25 Muscle Nerve 1986;9:809-19.

4 Jablecki CK. Single Fiber Electromyography. AAEE Minimonograph No. 6. Rochester, MN: American Association of Electromyography and Electrodiagnosis, 1978.

5 Sanders DB, Howard JF, Johns TR. Single-fiber electromyography in myasthenia gravis. Neurology 1979; 21:68-76.

6 Told-Hochstetter. Anatomischer Atlas fuer Studierende und Aerzte. Dritter Band. Nervensystem. 23. Auflage. Neubearbeited by Hayek $\mathbf{H}$. Wien-Innsbruck, Urban \& Schwarzenberg, 1960:124.

7 Stalberg E, Trontelj JV. Demonstration of axon reflexes in human motor nerve fibres. J Neurol Neurosurg Psychiatry 1970;33:571-9.

8 Trontelj JK, Mihelin M, Pletersek K, Antoni L. Jittermeter: a microcomputer-based system for single fibre electromyography. Int J Biomed Comput 1979;10: 451-9.

9 Trontelj JV, Mihelin M, Fernandez JM, Stalberg E. Axonal stimulation for end-plate jitter studies. $J$ Neurol Neurosurg Psychiatry 1986;49:677-85.

10 Stalberg E. Propagation velocity in human muscle fibres in situ. Acta Physiol Scand 1966;70, Suppl. 287:1-112.

11 Andreassen S, Arendt-Nielsen L. Muscle fibre conduction in motor units of the human anterior tibial muscle: A new size principle parameter. $J$ Physiol (Lond) 1987;391:561-71.

12 Stalberg E, Schiller HH, Schwartz MS. Safety factor in single human motor end-plates studied in vivo with single fibre electromyography. J Neurol Neurosurg Psychiatry 1975;38:799-804. 